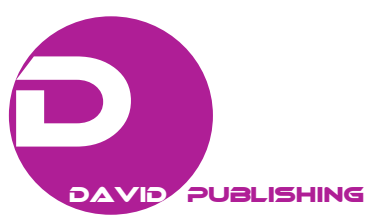

\title{
Electrical Brain Stimulation to Treat Neurological Disorder
}

\author{
Bahman Zohuri and David R. Modisette \\ Electrical Engineering and Computer Science Department, University of New Mexico, Albuquerque, New Mexico 87131, USA
}

\begin{abstract}
Neurological disorders with symptoms such as chronic pain, depression, and insomnia are widespread. Very weak electric fields applied through the skull can enhance or diminish neural activity and modulate brain waves in order to treat many of these common medical problems. This approach is to be contrasted with well-established pharmacological methods or more recent invasive electrical Deep Brain Stimulation (DBS) techniques that require surgery to insert electrodes deep into the brain. We claim that Non-Invasive Brain Stimulation (NIBS) will provide new treatment methods with much greater simplicity, lower cost, improved safety and in some cases, possibly greater effectiveness. This emerging use of NIBS is a branch of a new multidisciplinary field that we coined Neuro-systems Engineering [1]. This field involves neuroscientists, psychologists, and electrical engineers. This emerging field relies on existing standards for the safe implementation of these novel treatment modalities [2]. Methods of stimulating the brain are based on emerging electro-technologies such as transcranial Direct Current/Alternating Current (DC/AC) electric fields and pulsed magnetic fields. Application of functional and time-dependent brain imaging methods can be used to locate relevant brain regions and determine the most appropriate stimulation method. Application of tailored and individualized control can be combined with other therapy methods to effectively treat neurological disorders while minimizing or even eliminating the use of pharmaceuticals. In this paper, we are presenting our embodiment for a closed loop, feedback controlled, non-invasive application of electrical stimulation of the brain to enhance individual/group performance or to treat neurological disorders.
\end{abstract}

Key words: Repetitive Transcranial Magnetic Stimulation (rTMS), repetitive Transcranial Electric Stimulation (rTES), Deep Brain Stimulation (DBS), Electroencephalogram (EEG), Magnetoencephalography (MEG), transcranial Direct Current Stimulation (tDCS), transcranial Alternating Current Stimulation (tACS).

\section{Introduction}

The application of an electric field to the brain has been demonstrated for a variety of neurological conditions including the treatment of psychological disorders. However, many of the known methods involve invasive surgical procedures that carry considerable risk. While some noninvasive methodologies have begun to show promise, novel devices and methods are needed to address a variety of conditions for which electrotherapies can be helpful.

\section{Detailed Description}

According to various embodiments the present disclosure provides various devices and methods for

Corresponding author: Bahman Zohuri, Ph.D., associate professor, research fields: electrical and computer engineering. providing therapeutic and/or beneficial cranial electrostimulation. Accordingly, the present disclosure provides methods and apparatus that utilize a combination of real time brain functional monitoring and noninvasive electrical and/or magnetic trans-cranial brain stimulation to modify the brain function as exhibited in individual and group activities.

According to a specific embodiment, an Electroencephalogram (EEG), which is a technique of testing that is used to evaluate the electrical activity in the brain, where brain cells communicate with each other through electrical impulses (i.e. Fig. 1) or Magnetoencephalography (MEG), which is a non-invasive technique for investigating human brain. It allows the measurement of ongoing brain activity on a millisecond-by-millisecond basis, and it shows where in the brain activity is produced (i.e. Fig. 2). 


\section{Brain Waves: EEG Tracings}

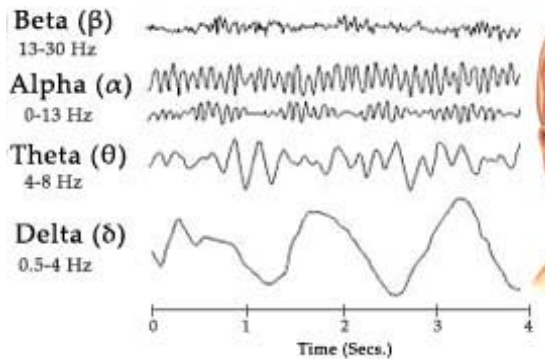

Fig. 1 Brain wave.

Source: www.medicin.net.

\section{Origins of MEG Signals}

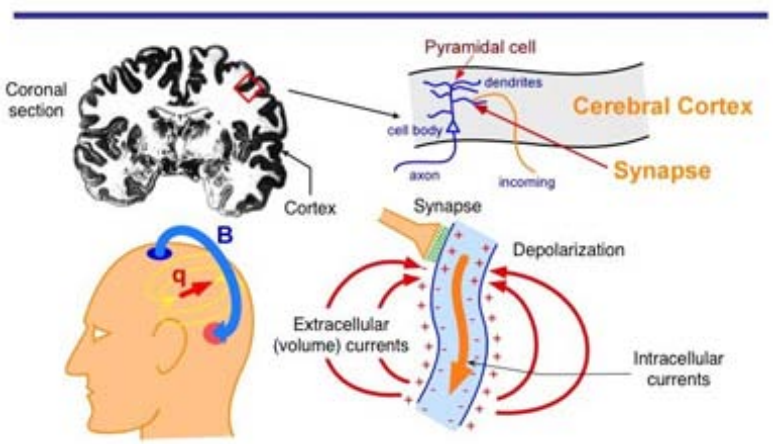

Fig. 2 Illustration of MEG origins.

Source:

http://ilabs.washington.edu/what-magnetoencephalography-me g.

In case of EEG a routine clinical Electroencephalography as it is illustrated in Fig. 3, recording typical lasts in about 20-30 minutes, which is called Pulse Preparation Time (PPT) and usually involves recording from scalp electrodes.

In routine clinical Electroencephalography, EEG, as illustrated in Fig. 3, the recording typically lasts 20 to 30 minutes and there is an additional time called Pules Preparation Time (PPT). The recording is made from scalp electrodes.

The MEG technique is a functional way of mapping brain activity by recoding magnetic fields that are driven by naturally occurring electrical currents in the brain. It uses very sensitive magnetometers as illustrated in Fig. 4.

According to a specific embodiment, generally speaking, an EEG or MEG device is used to measure and record the location, amplitude, and magnitude of time dependent electric and/or magnetic field oscillations of the brain. These oscillations indicate neural activity that can be related to normal functions such as the sleep cycle, pattern recognition, learning, communications and decision making. These signals can also be indicative of abnormal functions caused by sleep deprivation, stress, epilepsy, autism, addiction, and stress disorders

A device can measure these time dependent electric field oscillations and provide interpretation of the measurements, identify signals that are indicative of an abnormal or undesirable function, and generate a modified signal that can be transmitted into the brain using a trans-cranial brain stimulator such as transcranial Direct Current Stimulation (tDCS), transcranial Alternating Current Stimulation (tACS) or Transcranial Magnetic Stimulation (TMS) in order to produce a desired effect.

For example, if the detected brain output is indicative of the onset of an undesirable brain process, such as the initial stage of a seizure, then the signal

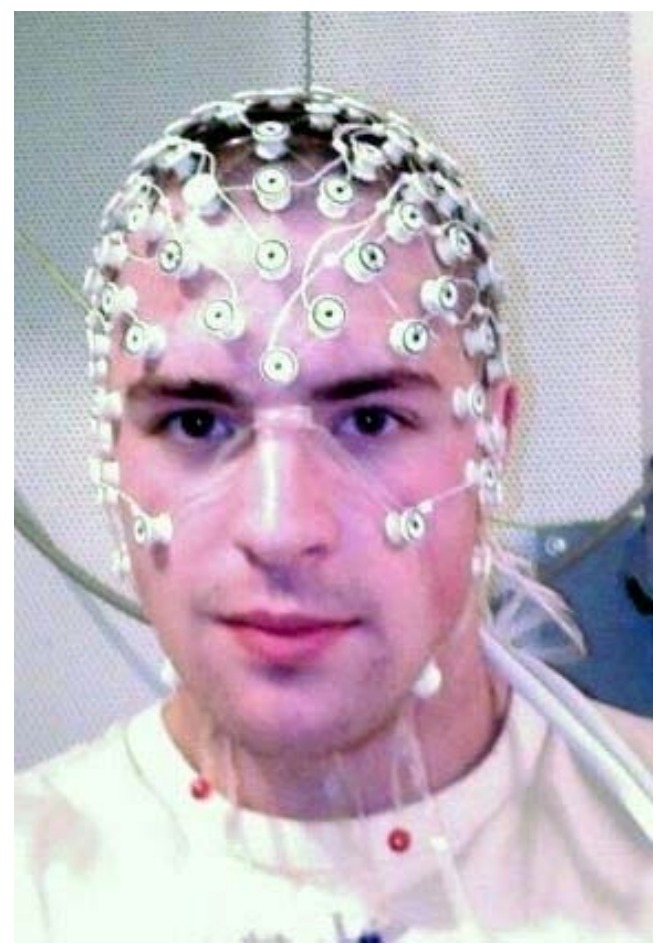

Fig. 3 Person undergoing an EEG recording setup. Source: www.wikipedia.com. 


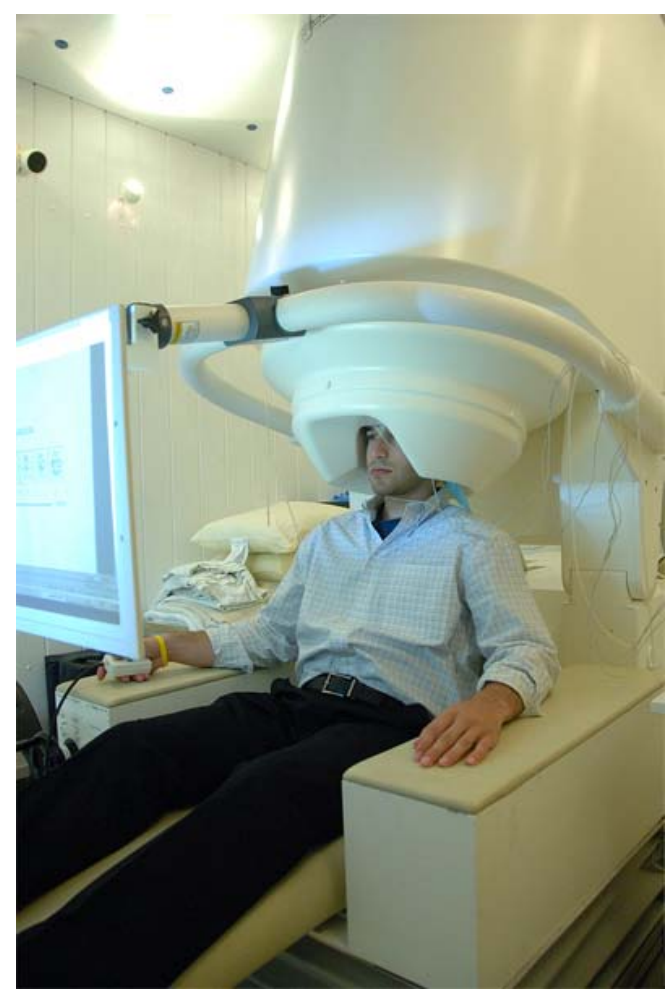

Fig. 4 Person undergoing an MEG process.

Source: www.wikipedia.com.

generated by the device and delivered to the brain would provide an in-phase, equal magnitude, but opposite sign in order to cancel that signal through destructive interference with the output signal. This superposition of an opposite sign signal is similar to the method of acoustic noise cancellation commonly used in active acoustic noise cancellation headphones. Accordingly, a unique feature of the inventions provided in this disclosure is the application of a "cancellation signal" within the brain region generating the undesirable output signal.

The application of a pulsed, oscillating, or DC electric field to modify neural activity is already being explored for treatment of depression [7], migraines [8], consolidating memory during slow wave sleep [9], and also in accelerated training of target detection [10].

These approaches apply a stimulus in an on/off manner based on a prescribed dose/time relationship. In stark contrast, according to various embodiments, the presently described invention utilizes closed loop feedback in order to provide active modification of a device-generated input signal in response to the brain's output signal.

According to an embodiment, a Feedback Device (FD) is connected to electrodes that are placed on the head in locations that are optimized for activation or deactivation of signals of interest that are produced by the brain. For instance, if the output is indicative of the early stage of a seizure in a localized brain region, the electrodes are located to provide or direct a cancellation wave to the part of the brain responsible for generating the early stage seizure-related signals in order to prevent the growth of wide spread coupled brain oscillations. According to various embodiments, at least a portion of the FD could take the form of a headset, cap, hat, helmet, head draping, or pillow.

According to yet another embodiment, the FD could be placed and optimized to encourage the brain to generate particular signals or cycles of signals. For example, a suitable application may be sleep deprivation caused by undesirable rapid transition from non-REM sleep into REM sleep. Here the purpose of the input field would be to entrain the signals produced by the brain that are associated with healthy sleep cycles and reduce the frequency of maladaptive patterns of sleep.

Note that: Rapid Eye Movement (REM) sleep is a kind of sleep that occurs at intervals during the night and is characterized by rapid eye movement, more dreaming and bodily movement, and faster pulse and breathing. See Fig. 5.

Bear in mind that your brain waves move fast, when you are awake. This is known as "beta" waves and when you lie down, close your eyes and become drowsy your brain wave tends to start slowing down; this is known as the "alpha" state. Alpha stage is consistent of 4 stages and each stage can be broken down to different mode five minutes into the alpha state, where your body slips into Stage 1 sleep [3].

Alternatively, the FD could be designed to encourage restorative slow wave sleep and prevent quick or 


\section{Typical Sleep Cycles}

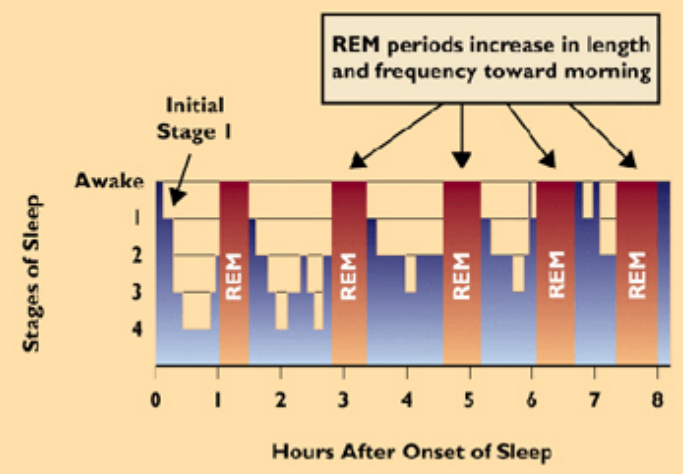

Fig. 5 Typical sleep cycles.

Source:

http://academic.pgcc.edu/ mhspear/sleep/stages/ltsleep.htm.

premature transition into REM sleep. Control of sleep brain patterns, either by preventing undesirable signals or by controlling the signal patterns over time could help reduce or prevent nightmares, and/or produce sleep that is more restorative over shorter durations, essentially allowing for an electrically stimulated powernap. Similar patterns could be profoundly useful for treatment of disorders such as Post-Traumatic Stress Disorder (PTSD) [11].

As stated above, the FD could be designed such that one or more electrodes are placed so as to direct the FD-generated signal towards those regions of the brain that are responsible for generating the signal of interest. In some embodiments, arrays of electrodes may be utilized to localize or concentrate FD-generated signals to one or more specific regions of the brain.

According to still another embodiment, the FD could be placed and optimized not to cancel an undesirable signal, but rather to amplify a desirable, naturally occurring signal. Alternatively, the FD could cancel or suppress some signals while amplifying others. For example, in applications to enhance memory, learning, or pattern recognition, the detection of a desirable signal would allow the FD to amplify that signal associated with storage of the information of interest separately or in concert with suppressing cognitive processes that compete for resources that could be used to encode memory.

According to yet another embodiment, two or more FDs could be in electrical communication with one another. In this embodiment, an FD of a first individual could transmit information to the FD of another individual or to the FDs of a group of individuals in order to enhance team performance by manipulating attention, engagement, and/or coordination of the group.

\section{Applications}

In a military application involved in a small group attempting to deal with ambiguous unstructured information, the coupling of multiple FDs would lead to enhanced detection of relevant information and coordination of the group. For example, if the FD of one member of a group identified brain waves associated with increased alertness, for example in response to the individual noticing "unusual or suspicious activity”, the FDs of the other members of the group could be programmed to increase alertness for all members of the group within a predetermined proximity.

According to another embodiment, the individual FDs could be coupled to remotely located computers to provide additional real time processing and memory for each of the FDs, and these computers could then be connected into a feedback and control system to provide overall management and coordination of the ensemble. For example, the FDs could be used to enhance the performance of a team of cyber defenders who are dealing with rapidly changing ambiguous information. The ability to detect pre-conscious patterns has been demonstrated [12], and the sharing of these preconscious detections would enhance the speed and coordination of the group. The ability to amplify this detection capability of the individuals and the group would lead to substantial performance enhancements.

According to yet another embodiment, rather than generating a signal that is equal and opposite to the 
signal of interest, the FD could introduce white noise so as to disrupt the signal of interest.

All patents and publications referenced or mentioned herein are indicative of the levels of competency of those skilled in the art to which the invention pertains, and each such referenced patent or publication is hereby incorporated by reference to the same extent as if it had been incorporated by reference in its entirety, individually, or set forth herein in its entirety. Applicants reserve the right to physically incorporate into this specification any and all materials and information from any such cited patents or publications. The specific methods and compositions described herein are representative of preferred embodiments and are exemplary and not intended as limitations on the scope of the invention. Other objects, aspects, and embodiments will occur to those skilled in the art upon consideration of this specification and are encompassed within the spirit of the invention as defined by the scope of the claims. It will be readily apparent to one skilled in the art that varying substitutions and modifications may be made to the invention disclosed herein without departing from the scope and spirit of the invention.

The invention illustratively described herein suitably may be practiced in the absence of any element or elements, or limitation or limitations, which is not specifically disclosed herein as essential. The methods and processes illustratively described herein suitably may be practiced in differing orders of steps, and that they are not necessarily restricted to the orders of steps indicated herein or in the claims. As used herein and in the appended claims, the singular forms "a”, "an", and "the” include plural reference unless the context clearly dictates otherwise. Thus, for example, a reference to "a host cell" includes a plurality (for example, a culture or population) of such host cells, and so forth.

Under no circumstances may the patent be interpreted to be limited to the specific examples or embodiments or methods specifically disclosed herein.
The terms and expressions that have been employed are used as terms of description and not of limitation, and there is no intent in the use of such terms and expressions to exclude any equivalent of the features shown and described or portions thereof, but it is recognized that various modifications are possible within the scope of the invention as claimed. Thus, it will be understood that although the present invention has been specifically disclosed by preferred embodiments and optional features, modification and variation of the concepts herein disclosed may be resorted to by those skilled in the art, and those such modifications and variations are considered to be within the scope of this invention as defined by the appended claims.

The invention has been described broadly and generically herein. Each of the narrower species and sub-generic groupings falling within the generic disclosure also forms part of the invention. This includes the generic description of the invention with a proviso or negative limitation removing any subject matter from the genus, regardless of whether or not the excised material is specifically recited herein.

\section{What Is Claimed?}

What is claimed here, is summarized as follow as a conclusion for this paper and they are:

- A non-invasive device for controlling neuronal activity in the brain comprising a Feedback Device (FD);

- A sensor for detecting an electrical signal of interest generated by the brain;

- A means for generating an electrical signal;

- A means for delivering the general electrical signal to the brain, and;

- A processor such as Tensor Central Processing Unit (TPU) or Graphic Processing Unit (GPU) configured to identify an electrical signal of interest generated by the brains and direct a corresponding cancellation signal to the region of the brain responsible for generation the electrical signal of interest. 
The above conclusion is falling to the processors that they have a cognitive and image processing for deep machine learning drive by Supper Artificial Intelligence (SAI) that is published by Zohuri et. al. [4-6] and they may act as a "MATRIX" of near future.

\section{References}

[1] Yonas, G., and Jung, R. 2008. "Fixing brains.”Innovation: America's Journal of Technology Commercialization 6(3). Available at http://www.innovationamerica.org/fixing-brains.

[2] Wassermann, E.M. 1998. "Risk and Safety of Repetitive Transcranial Magnetic Stimulation: Report and Recommendations from the International Workshop on the Safety of Repetitive Transcranial Magnetic Stimulation, June 5-7, 1996.” Electroenceph. Clin. Neurophysiol. 108: 1-16.

[3] Terry,

B. "Light/Deep Sleep.” http://academic.pgcc.edu/ mhspear/sleep/stages/lt sleep.html.

[4] Zohuri, B., and Moghaddam, M. 2018. “Artificial Intelligence Driven by a General Neural Simulation System-Genesis.” Jan. 29, 2018, Nova Science Publication Inc. Series: Neurology-Laboratory and Clinical research Developments。
[5] Zohuri, B., and Moghaddam, M. 2017. "Neural Network Driven Artificial Intelligence: Decision Making Based on Fuzzy Logic (Computer Science, Technology and Applications: Mathematics Research Developments).” Jul. 24, 2017, Nova Science Publication Inc. Series: Computer Science, Technology and Applications: Mathematics Research Developments.

[6] Zohuri, B., and Moghaddam, M. 2018. “Neural Network Driven Supper Artificial Intelligence Based on Internet of Things and Big Data." SciFed Journal of Artificial Intelligence 1 (1): 1-24.

[7] Nitsche, M. A., Boggio, P. S., Fregni, F., and Pascual-Leone, A. Sept., 2009. "Treatment of Depression with tDCS: A Review”. Exper Neurol Vol. 219.

[8] U "SCSF Med Center, April, 2009, New Magnetic Stimulation Therapy Shows Promise as Migraine Therapy."

[9] Marshall, L., Mölle, M., Hallschmid, M., and Born, J. 2004. "tDCS During Sleep Improves Declarative Memory.” Journal of Neuroscience, Nov. 2004.

[10] Gerold Yonas, PhD, private communication, March 2018.

[11] Krakow, B., "Sleep Disorders Co-morbidity Theory: A Novel Approach to PTSD Prevention and Treatment." Neuroscience for National Security Symposium, May 2010.

[12] DARPA Pursues Neuroscience to Enhance Analyst, “Soldier Performance”. Av. Week, Jan. 2008. 